\title{
Die Bearbeiter der 4. Auflage
}

Marc von Ammon, LL.M., Rechtsanwalt/Fachanwalt für Bank- und Kapitalmarktrecht, DWF Germany Rechtsanwaltsgesellschaft mbH, Köln

Prof. Dr. Heribert M. Anzinger, Universität Ulm

Dr. Volker Baas, Rechtsanwalt, Taylor Wessing Partnerschaftsgesellschaft mbB, Frankfurt am Main

Damir Barac, Wirtschaftsprüfer, GAR Gesellschaft für Aufsichtsrecht und Revision mbH WPG, Frankfurt am Main

Dr. Jürgen Baur, Rechtsanwalt, AACHENER GRUNDVERMÖGEN Kapitalverwaltungsgesellschaft mbH, Köln

Dr. Caspar Behme, Universität Heidelberg/LMU München

Dr. Florian Bentele, Rechtsanwalt, Eschborn/Syndikusrechtsanwalt bei Allianz Global Investors GmbH, Frankfurt am Main

Bastian Bubel, Rechtsanwalt, Lampertheim

Lidija Delčev, Diplom-Ökonomin, Ernst \& Young GmbH, Eschborn

Manfred Dietrich, Rechtsanwalt, Norton Rose Fulbright Luxembourg SCS, Luxembourg

Nico Dorenkamp, Rechtsanwalt/Steuerberater, Vistra GmbH \& Co. KG Wirtschaftsprüfungsgesellschaft, München

René Dubois, Rechtsanwalt, Dentons Europe LLP, München

Katrin Ebel, Volljuristin, Hannover

David Eckner, LL.M. (KCL), Universität Düsseldorf

Dr. Björn Enders, Rechtsanwalt/Fachanwalt für Steuerrecht/Steuerberater/Diplom-Finanzwirt (FH)/ Licencié en Droit (Paris II), DLA Piper UK LLP, Frankfurt am Main

Anne Engelhardt, Kaiserslautern

Liliane Feller, Rechtsanwältin, München

Marc Fellner, Rechtsanwalt/Steuerberater, DLA Piper UK LLP, Frankfurt am Main

Andreas Fiand, Diplom Finanzwirt, Oberfinanzdirektion Karlsruhe

Juliane Fitzke, Rechtsanwältin, Taylor Wessing Partnerschaftsgesellschaft mbB, Berlin

Dr. Burkhard Führmeyer, Rechtsanwalt, DLA Piper UK LLP, Frankfurt am Main

Thomas Geese, Steuerberater, Société Générale Securities Services GmbH, Unterföhring

Finn Gerlach, Rechtsanwalt, Deutscher Sparkassen- und Giroverband e.V., Berlin

Dr. Matthias Geurts, Rechtsanwalt, Noerr LLP, Frankfurt am Main

Dr. Harald Glander, LL.M., Rechtsanwalt, Simmons \& Simmons LLP, Frankfurt am Main

Lissie Goldbach, Rechtsanwältin, Baker McKenzie LLP, Luxembourg

Jens Grimm, Wirtschaftsprüfer/Steuerberater, München

Tim Hackemann, Rechtsanwalt/Steuerberater, Ernst \& Young GmbH, Eschborn

Alexander Hagen, Rechtsanwalt/Steuerberater, Clifford Chance Deutschland LLP, Frankfurt am Main

Dr. Mathias Hanten, M.B.L., Rechtsanwalt, Deloitte Legal Rechtsanwaltsgesellschaft mbH, Frankfurt am Main

Sebastian Hartrott, Rechtsanwalt, HANNOVER LEASING GmbH \& Co. KG, Pullach

Dr. Carlo Heck, Rechtsanwalt, Lapithus Management S.à.r.l., Luxembourg

Martin Heinsius, Rechtsanwalt/Steuerberater, DLA Piper UK LLP, Frankfurt am Main

Tom Herberger, Referent im Bundesministerium der Justiz und für Verbraucherschutz, Berlin

Frank Herring, Rechtsanwalt, Allen \& Overy LLP, Frankfurt am Main

Dr. Florian Herrmann, Rechtsanwalt/Fachanwalt für Handels- und Gesellschaftsrecht, Langwieser

Rechtsanwälte Partnerschaft, München, Berlin

Nadine Hesser, Rechtsanwältin/Fachanwältin für Arbeitsrecht, DLA Piper UK LLP, Frankfurt am Main

Timo Hillebrand, Diplom Wirtschaftsjurist (FH), Ernst \& Young GmbH, Eschborn

Prof. Dr. Fabian Hinrichs, Rechtsanwalt, DLA Piper UK LLP, Frankfurt am Main

Dr. Daniel Hoffmann, Bundesverband Deutscher Banken e.V., Berlin

Martin Hüwel, Rechtsanwalt, Luther Rechtsanwaltsgesellschaft mbH, Frankfurt am Main

Dr. Anna Lucia Izzo-Wagner, LL.M. Eur., Rechtsanwältin, Taylor Wessing Partnerschaftsgesellschaft mbB, Frankfurt am Main

Christof Gerhard Jenett, Rechtsanwalt/Steuerberater, Ernst \& Young GmbH, Eschborn

Dr. Thomas A. Jesch, Bundesverband Institutioneller Investoren e.V., Frankfurt am Main 
Dirk Jessen, Steuerberater/Wirtschaftsprüfer, Mazars GmbH \& Co. KG, Hamburg

Dr. Matthias Josek, DWS Investment GmbH, Frankfurt am Main

Valeska Karcher, LL.M., Rechtsanwältin, Allen \& Overy LLP, Frankfurt am Main

Dr. Bert Kimpel, Rechtsanwalt/Steuerberater/Fachanwalt für Steuerrecht, Taylor Wessing Partnerschaftsgesellschaft mbB, Frankfurt am Main

Dr. Jens Kirchner, Rechtsanwalt, DLA Piper UK LLP, Frankfurt am Main

Dr. Ulf Klebeck, Rechtsanwalt

Christian Klein, Steuerberater/Wirtschaftsprüfer, VISTRA Treuhand GmbH Wirtschaftsprüfung, Köln

Dr. Fabian Klein, Rechtsanwalt, Ashurst LLP, Frankfurt am Main

Dr. Florian Kloster, Assistenzprofessor am Lehrstuhl für Betriebswirtschaftliche Steuerlehre, Internationales und Liechtensteinisches Steuerrecht, Universität Liechtenstein, Vaduz

Nils König, Steuerberater, Rechtsanwalt, Peine

Dr. Stefan Königer, Steuerberater, Ernst \& Young GmbH, Stuttgart

Dr. Jan Könnecke, Rechtsanwalt, Könnecke Naujok, Frankfurt am Main

Jannes Kracke, Rechtsanwalt, HANSAINVEST Hanseatische Investment-GmbH, Hamburg

Lukas Krönke, Rechtsanwalt, Langwieser Rechtsanwälte, München

Andreas B. Lammel, Rechtsanwalt, Kas Bank, Frankfurt am Main

Dr. Florian Leclerc, Verwaltungsgericht Sigmaringen

Peter Lenz, Wirtschaftsprüfer, VISTRA Treuhand GmbH Wirtschaftsprüfung, Köln

Karin Lichtenstein, Rechtsanwältin, Schiffdorf

Norman Mayr, LL.M., Rechtsanwalt, P+P Pöllath + Partners, Frankfurt am Main

Elnaz Mehrkhah, Rechtsanwältin, Frankfurt am Main

Horst Mertes, Rechtanwalt, Steuerberater, Frankfurt am Main

Jens Moericke, Rechtsanwalt, Frankfurt am Main

Dr. Sascha Morgenroth, LL.M., Rechtsanwalt, Fachanwalt für Arbeitsrecht, Simmons \& Simmons LLP, Frankfurt am Main

Nic Müller, Steuerberater/Wirtschaftsprüfer, KPMG Luxembourg, Société coopérative

Andrea München, LL.M., Rechtsanwältin/Avocat à la Cour, CMS Hasche Sigle, Frankfurt am Main/ Luxembourg

Dr. Markus München, LL.M., Rechtsanwalt/Steuerberater, Allen \& Overy LLP, München

Jürgen Nagler, Rechtsanwalt/Steuerberater, KPMG AG Wirtschaftsprüfungsgesellschaft, Frankfurt am Main

Dr. Robert Oppenheim, Rechtsanwalt/Fachanwalt für Bank- und Kapitalmarktrecht, lindenpartners, Berlin

Till Otto, Rechtsanwalt, Taylor Wessing Partnerschaftsgesellschaft mbB, Frankfurt am Main

Andreas Patzner, Rechtsanwalt/Steuerberater, KPMG AG Wirtschaftsprüfungsgesellschaft, Frankfurt am Main

Petra Reinholz, Rechtsanwältin, Heussen Rechtsanwaltsgesellschaft mbH, Berlin

Stephan Reiss, Rechtsanwalt, Ernst \& Young Law GmbH, Frankfurt am Main

Dr. Maximilian von Rom, Rechtsanwalt, Gleiss Lutz, Frankfurt am Main

Dr. Peter Schäfer, Rechtsanwalt/Fachanwalt für Steuerrecht, Fachkanzlei Schäfer, Commerzbank AG, Hanau/Frankfurt am Main

Christian Schinzl, Steuerberater/Wirtschaftsprüfer, Frankfurt am Main

Christoph Schmid, Rechtsanwalt, Würzburg

Ingolf Schneider-Deters, Rechtsanwalt/ Steuerberater, Nuveen Real Estate, Frankfurt am Main

Leif Schubert, Mag. rer. publ., Rechtsanwalt, Allen \& Overy LLP, Frankfurt am Main

Dr. Tilman Schultheiß, Rechtsanwalt, Thümmel, Schütze \& Partner, Dresden/Frankfurt am Main

Dr. Oliver von Schweinitz, Rechtsanwalt/Steuerberater/Fachanwalt für Steuerrecht/Attorney-at-Law

(New York), LPA-GGV Partnerschaftsgesellschaft, Frankfurt am Main

Dr. Nina-Luisa Siedler, Rechtsanwältin, DWF Germany Rechtsanwaltsgesellschaft mbH, Berlin

Sabrina Siemko, Rechtsanwältin, Darmstadt

Dr. Lea Maria Siering, Rechtsanwältin, Taylor Wessing Partnerschaftsgesellschaft mbB, Berlin

Peggy Steffen, Rechtsanwältin, Frankfurt am Main

Prof. Dr. Falko Tappen, Steuerberater, Hochschule Worms-University of Applied Sciences

Dr. Sebastian Taschke, Rechtsanwalt, Hauck \& Aufhäuser Privatbankiers AG, Frankfurt am Main 
Marcel Tschatsch, Wirtschaftsprüfer/Steuerberater, Ernst \& Young GmbH Wirtschaftsprüfergesellschaft, München

Sebastian Tusch, Rechtsanwalt, Frankfurt am Main

Ani Valcheva, Wissenschaftliche Mitarbeiterin, Deloitte Legal Rechtsanwaltsgesellschaft mbH

Dr. Christopher Virreira Winter, Rechtsanwalt, Dalli Group, Stolberg

Daniel Wahn, Richter am Landgericht in Bad Kreuznach

Simon Weber, Rechtsanwalt, Berlin

Frank Wiese, Rechtsanwalt, KPMG AG Wirtschaftsprüfungsgesellschaft, Frankfurt am Main

Christopher Winter, Martin-Luther-Universität Halle Wittenberg

Katja Simone Wülfert, Rechtsanwältin, Société Générale Securities Services GmbH, Unterföhring

Martin Zackor, Rechtsanwalt, Luther Rechtsanwaltsgesellschaft mbH, Frankfurt am Main

Heinz Zimmermann, Rechtsanwalt/Wirtschaftsprüfer/Steuerberater, DLA Piper UK LLP of Counsel, Köln

Dr. Frank Zingel, Rechtsanwalt, lindenpartners, Berlin

Marco Zingler, Rechtsanwalt/Dipl.-Finanzwirt (FH), Allen \& Overy LLP, Frankfurt am Main 
